\title{
Endogenous Monocarboxylates Sustain Hippocampal Synaptic Function and Morphological Integrity during Energy Deprivation
}

\author{
Yukitoshi Izumi, ${ }^{1}$ Ann M. Benz,, ${ }^{1}$ Hiroshi Katsuki, ${ }^{1}$ and Charles F. Zorumski ${ }^{1,2}$ \\ Departments of ${ }^{1}$ Psychiatry and ${ }^{2}$ Neurobiology, Washington University School of Medicine, St. Louis, Missouri 63110
}

The ability to fuel neurons via glycogenolysis is believed to be an important function of glia. Indeed, the slow, rather than immediate, depression of synaptic transmission in hippocampal slices during exogenous glucose deprivation suggests that intrinsic energy reservoirs help to sustain neurotransmission. It is believed that glia fuel neighboring neurons via diffusible monocarboxylates such as pyruvate and lactate, although a role for glucose has been proposed also. Using $\alpha$-cyano-4hydroxycinnamate (4-CIN) to inhibit monocarboxylate transport and cytochalasin $\mathrm{B}(\mathrm{CCB})$ to inhibit glucose transport, we examined the role of glucose and monocarboxylates in supporting the functional and morphological integrity of hippocampal neurons during glucose deprivation. Although $200 \mu \mathrm{M} 4-\mathrm{CIN}$ failed to depress EPSPs supported by $10 \mathrm{~mm}$ glucose, pretreatment with 4-CIN accelerated the depression of EPSPs during glucose deprivation. 4-CIN also accelerated the decline in glucose-supported EPSPs after administration of $50 \mu \mathrm{M}$ CCB, whereas CCB failed to alter the slow decay of pyruvatesupported EPSPs during pyruvate deprivation. 4-CIN did not alter the morphology of pyramidal neurons in the presence of $10 \mathrm{~mm}$ glucose but produced significant damage during glucose deprivation or CCB administration. These results suggest that endogenous monocarboxylates rather than glucose maintain neuronal integrity during energy deprivation. Furthermore, EPSPs supported by 2-3.3 mm glucose were sensitive to 4-CIN, suggesting that endogenous monocarboxylates are involved in maintaining neuronal function even under conditions of mild glucose deprivation.

Key words: hippocampus; energy metabolism; glucose transport; pyruvate; lactate; neuroglial interactions
The brain requires carbohydrate metabolism to meet its energy demands. The high rate of glycogen turnover in the brain (Siesjo, 1978) suggests that glycogen serves as an energy source when glucose supply is insufficient (Lowry et al., 1964). Thus, regional differences in glycogen content may account in part for regional differences in vulnerability to hypoglycemic insults (Sagar et al., 1987; Garriga et al., 1994). Because glycogen is stored in glia and little is present in neurons (Rosenberg and Dichter, 1985; Kato et al., 1989; Ignacio et al., 1990), energy substrates from glycogen breakdown must be supplied to neurons in forms that cross cell membranes. Potential energy sources include glucose and the monocarboxylates, pyruvate and lactate (Rust, 1994; Forsyth, 1996; Magistretti and Pellerin, 1996; Tsacopoulos and Magistretti, 1996).

Although glucose-6-phosphatase activity is low in the brain (Sokoloff et al., 1977; Dringen and Hamprecht, 1992) and it long has been believed that the brain cannot generate endogenous glucose (Scrutton and Utter, 1968), some findings suggest cerebral gluconeogenic activity (Prasannan and Subramanyam, 1968; Bhattacharya and Datta, 1993). Furthermore, it has been shown that astroglia release glucose extracellularly (Cambray-Deakin et al., 1988; Forsyth et al., 1996). Other studies, however, have

Received May 6, 1997; revised Sept. 9, 1997; accepted Sept. 30, 1997.

This work was supported by Grants from the Diabetes Research and Training Center at Washington University, Alzheimer's Disease and Related Disorders Program at the University of Missouri, National Institute of Mental Health (MH00964 and MH45493), and National Institute of Aging (AG11355) and fellowships from the Human Frontier Sciences Program and Bantly Foundation. We dedicate this work to the memory of Dr. Julio Santiago, who provided support and helpful discussions.

Correspondence should be addressed to Dr. Yukitoshi Izumi, Department of Psychiatry, Washington University School of Medicine, 4940 Children's Place, St. Louis, MO 63110.

Copyright (C) 1997 Society for Neuroscience $\quad 0270-6474 / 97 / 179448-10 \$ 05.00 / 0$ shown that lactate, but not glucose, is detected in the media of astroglia-rich cultures during glucose deprivation (Walz and Muckerji, 1988; Dringen et al., 1993a). Additionally, lactate has been proposed to be the substrate for glial-neuronal energy buffering in the retina and brain (Dringen et al., 1993c; Tsacopoulos and Magistretti, 1996). Lactate maintains synaptic function in the absence of glucose in hippocampal slices (Schurr et al., 1988) and can sustain cognitive function during hypoglycemia (Maran et al., 1994). A related energy substrate, pyruvate, also maintains synaptic activity and neuronal morphology during glucose deprivation or during administration of iodoacetate (IA), a glycolytic inhibitor (Izumi et al., 1994).

At present it remains unclear which energy substrates are provided by glia and used by neurons. Because glucose use requires energy expenditure whereas monocarboxylate use depends on respiration, the nature of the energy substrate is important, particularly during ischemia in which there are both energy deprivation and hypoxia. In the present study we examined whether glucose or glycolytic intermediates support synaptic function during glucose deprivation, using 4-CIN to inhibit monocarboxylate transport and CCB to inhibit glucose transport (Dringen et al., 1993c; Fowler, 1993; Williams et al., 1996). Hypothetical pathways for glial-neuronal interaction and the effects of 4-CIN and CCB are presented as diagrams in Figures 1, 3-6.

\section{MATERIALS AND METHODS}

Slices were prepared from the septal half of the hippocampus by standard techniques (Zorumski et al., 1996). Albino rats $(30 \pm 2 \mathrm{~d}$ old) were anesthetized with halothane and decapitated. The hippocampi were dissected rapidly and placed in artificial CSF (ACSF) containing (in $\mathrm{mm}$ ): $124 \mathrm{NaCl}, 5 \mathrm{KCl}, 2 \mathrm{MgSO}_{4}, 2 \mathrm{CaCl}_{2}, 1.25 \mathrm{NaH}_{2} \mathrm{PO}_{4}, 22 \mathrm{NaHCO}_{3}$, and 10 glucose, bubbled with $95 \% \mathrm{O}_{2} / 5 \% \mathrm{CO}_{2}$ at $4-6^{\circ} \mathrm{C}$, and sliced transversely into $500 \mu \mathrm{m}$ slices with a World Precision Instruments vibroslicer (Sarasota, FL). Then the slices were placed in an incubation 

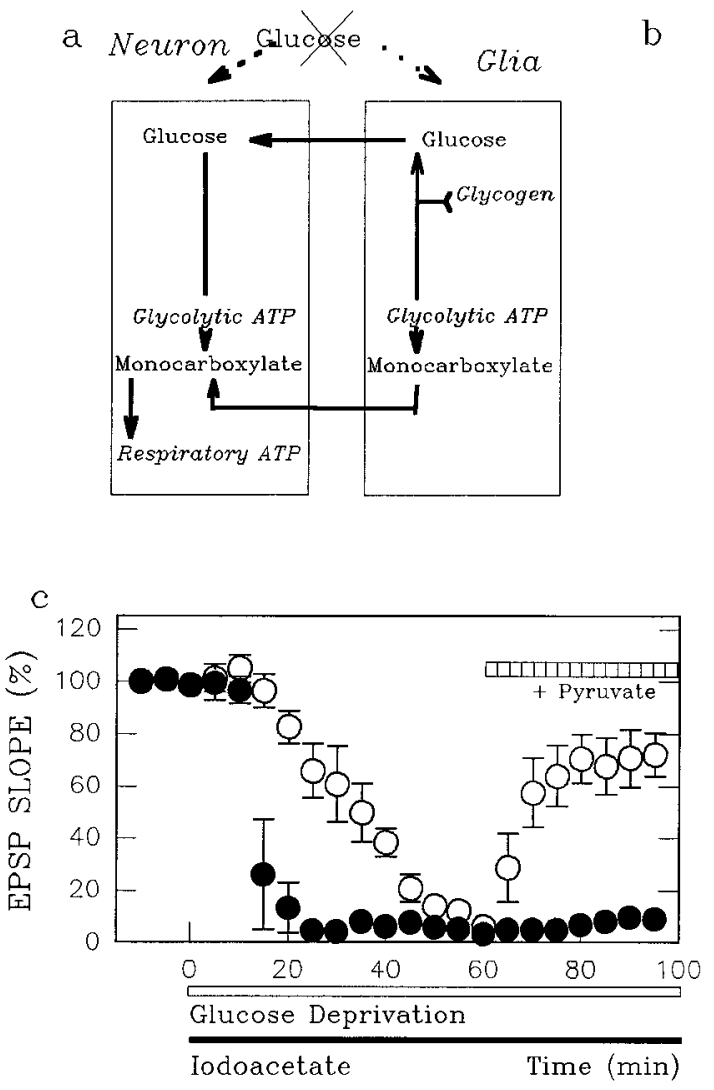

b

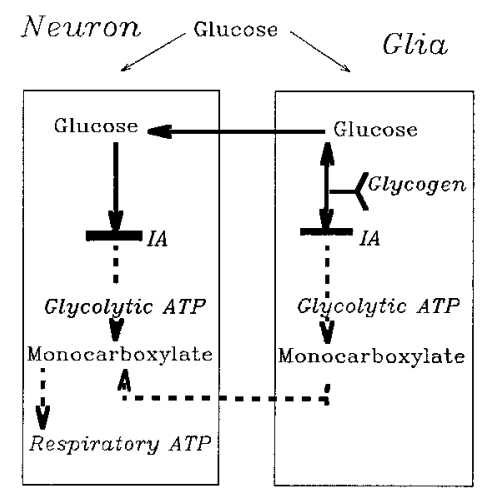

Glucose

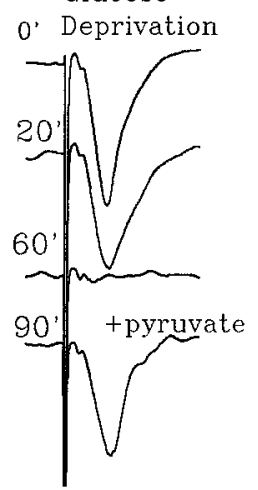

Iodoacetate

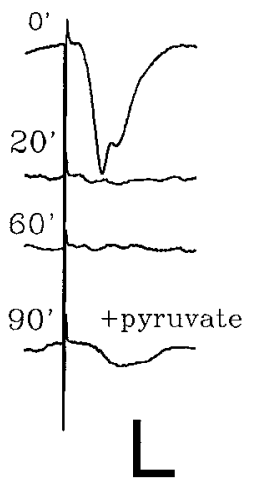

Figure 1. Evidence for existence of endogenous energy buffer. $a$, Presented is a scheme for glial-neuronal interaction during glucose deprivation. During exogenous glucose deprivation, glial glycogen may support neurons by providing glucose or monocarboxylates. $b$, Iodoacetate (IA) has effects that differ from glucose deprivation. Regardless of the energy source used in glial-neuronal interactions, the energy buffering system is not functional in the presence of IA, which inhibits glycolysis at glyceraldehyde-3phosphate dehydrogenase (GAPDH) in both types of cells. $c$, Glucose-supported EPSPs (open circles) decay slowly during glucose deprivation (open bar) and are restored by $10 \mathrm{~mm}$ pyruvate introduced 60 min after glucose deprivation (hatched bar). In contrast, $200 \mu \mathrm{M}$ IA (filled bar) suppresses EPSPs promptly (filled circles). Although IA does not suppress EPSPs in the presence of pyruvate (Izumi et al., 1994), adding $10 \mathrm{~mm}$ pyruvate $60 \mathrm{~min}$ after IA administration poorly restores synaptic activity. The traces on the right are representative field EPSPs in a slice treated with glucose deprivation (left $\mathrm{col}$ umn of traces) and in a slice treated with IA (right column). The number on each trace denotes the time (in minutes) when the response was obtained in the graph at left. Calibration bar, $1 \mathrm{mV}, 5 \mathrm{msec}$. chamber containing gassed $\mathrm{ACSF}$ for $1 \mathrm{hr}$ at $30^{\circ} \mathrm{C}$. At the time of study, slices were transferred individually to a submersion recording chamber. Experiments were done at $30^{\circ} \mathrm{C}$.

Extracellular recordings were obtained from the dendritic region of CA1 for analysis of population EPSPs, using $2 \mathrm{~m} \mathrm{NaCl}$ glass electrodes with resistances of 5-10 $\mathrm{M} \Omega$. Evoked synaptic responses were elicited with $0.1-0.2 \mathrm{msec}$ constant current pulses through a bipolar electrode placed in the Schaffer collateral-commissural pathway. After establishing a stable baseline for at least $10 \mathrm{~min}$ and a control input-output (IO) curve, we monitored synaptic responses by applying single stimuli to the Schaffer collateral pathway every $60 \mathrm{sec}$ at an intensity sufficient to elicit a 50-60\% maximal EPSP. IO curves were repeated 20 min after drug administration, 20 and $60 \mathrm{~min}$ after energy deprivation, and $30 \mathrm{~min}$ after reperfusion with energy substrates.

All chemicals were obtained from Sigma (St. Louis, MO). Drugs were dissolved in the ACSF and administered by bath perfusion. Experiments that used pyruvate or lactate were started $30 \mathrm{~min}$ after switching from glucose-containing ACSF. Pyruvate and lactate were used in combination with changes in $\mathrm{NaCl}$ to maintain osmolarity. All data are expressed as mean \pm SEM as compared with the initial control values. Differences between controls and experimental groups were assessed with nonparametric statistics.

For histological experiments, hippocampal slices from the same animal were incubated in parallel in individual $10 \mathrm{ml}$ beakers at $30^{\circ} \mathrm{C}$. Each hippocampus provided six to eight slices, and experimental and control experiments were run simultaneously on slices prepared from the same animal. At the completion of an experiment, slices were fixed in $1 \%$ paraformaldehyde and $1.5 \%$ glutaraldehyde overnight at $4{ }^{\circ} \mathrm{C}$. Then the slices were rinsed in $0.1 \mathrm{M}$ pyrophosphate buffer, placed in $1 \%$ buffered osmium tetroxide for $60 \mathrm{~min}$, and dehydrated with alcohol and toluene. Slices were embedded in Araldite, cut into $1 \mu \mathrm{m}$ sections, stained with methylene blue and azure II, and evaluated by light microscopy. Damage in the CA1 region was rated on a 0 (completely intact) to 4 (severe damage with dissolution of pyramidal neurons) scale by a rater who was unaware of the experimental condition. Using this system, we rated control slices that were incubated for $120 \mathrm{~min}$ in standard ACSF as $0.2 \pm$ $0.1(n=34)$. Slices treated with $200 \mu \mathrm{M}$ IA for $90 \mathrm{~min}$ (Izumi et al., 1994) or $20 \mathrm{~min}$ of simulated ischemia (anoxia plus no glucose), followed by 90 min postincubation in standard ACSF (Izumi et al., 1996), typically were rated as 4 .

\section{RESULTS}

\section{Differences between glucose deprivation and glycolytic inhibition}

As shown previously (Schurr et al., 1988; Fowler, 1993; Izumi et al., 1994), glucose deprivation produced a slowly developing depression of synaptic responses in the CA1 region of hippocampal slices, with complete elimination of synaptic transmission requiring $>30 \mathrm{~min}$. After $60 \mathrm{~min}$ of glucose deprivation, EPSPs were restored to $73.6 \pm 6.5 \%$ of control $(n=5)$ by administration of 10 $\mathrm{mm}$ pyruvate in place of glucose (open circles in Fig. 1c), indicating that neurons are able to use monocarboxylates to sustain function in the absence of glucose. Although the depression of EPSPs by glucose deprivation occurred slowly, IA, an inhibitor of glyceraldehyde-3-phosphate dehydrogenase (GAPDH), depressed EPSPs much more quickly (Schurr et al., 1988; Izumi et al., 1994). During $20 \mathrm{~min}$ of glucose deprivation, EPSPs were depressed to $66.6 \pm 6.1 \%$ of control $(n=5)$, whereas a $20 \mathrm{~min}$ administration of $200 \mu \mathrm{M}$ IA suppressed EPSPs to $9.0 \pm 1.7 \%$ of control $(n=5 ; p<0.01)$ ( filled circles in Fig. $1 c)$. The difference in the rate of depression of synaptic responses during glucose deprivation and IA treatment suggests that endogenous energy buffers that normally are metabolized by the glycolytic pathway sustain synaptic function during energy source deprivation. Although IA does not suppress EPSPs in the presence of $10 \mathrm{~mm}$ pyruvate (Izumi et al., 1994), perfusion of $10 \mathrm{~mm}$ pyruvate $60 \mathrm{~min}$ after IA administration failed to restore EPSPs $(10.4 \pm 2.0 \%$ control; $n=5$ ). 

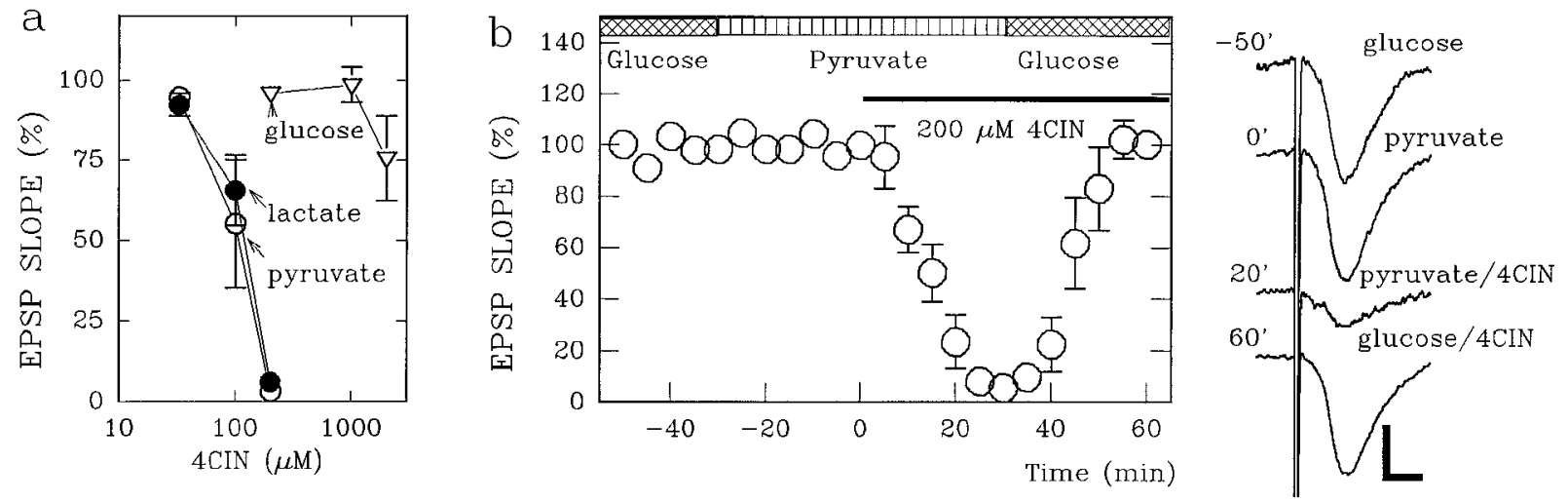

Figure 2. Selective inhibition of monocarboxylate-supported EPSPs by 4-CIN. a, Shown are dose-response curves for the effect of 4-CIN on EPSP slopes supported by $10 \mathrm{~mm}$ pyruvate (open circles), lactate ( filled circles), and glucose (open triangles). 4-CIN was perfused for 20 min before obtaining input-output curves of EPSP slopes ( $n=4$ for each point). $b$, EPSPs are not depressed by switching the energy substrate in the media from 10 mM glucose (cross-hatched bar) to $10 \mathrm{~mm}$ pyruvate (striped bar). Although administration of $200 \mu \mathrm{M}$ 4-CIN ( filled bar) suppresses pyruvate-supported EPSPs, administration of glucose in the presence of 4-CIN restores EPSPs. The traces on the right are representative field EPSPs in a slice sampled before and $30 \mathrm{~min}$ after switching the energy substrate to pyruvate, $20 \mathrm{~min}$ after 4-CIN administration, and $30 \mathrm{~min}$ after switching back to glucose. The number on each trace denotes the time (in minutes) when the response was obtained in the graph. Calibration bar, $1 \mathrm{mV}, 5 \mathrm{msec}$.

\section{Effects of monocarboxylate and glucose transport inhibitors on pyruvate- and glucose-supported EPSPs}

If glial glycogen serves as the source of energy to sustain synaptic function in the absence of exogenous glucose, then the buffering energy source must be transferred to neurons as glucose or monocarboxylates. Glucose and the monocarboxylates use different transporters that are inhibited by CCB (Fowler, 1993) and 4-CIN (Cox et al., 1985; Williams et al., 1996), respectively, making it possible to determine which form of energy substrate is used by neurons during energy deprivation. To examine the specificity of CCB and 4-CIN on glucose and monocarboxylate transporters, we examined the effects of these agents on synaptic transmission sustained by glucose, pyruvate, or lactate. EPSPs supported by $10 \mathrm{~mm}$ pyruvate (pyruvate-supported EPSPs) were depressed readily by $200 \mu \mathrm{M} 4-\mathrm{CIN}$ (EPSP slopes, $23.3 \pm 10.3 \%$ and $2.3 \pm 1.8 \%$ of control at 20 and 30 min after $4-\mathrm{CIN} ; n=5$ ). These synaptic responses were restored to $99.9 \pm 4.3 \%$ of control by administration of $10 \mathrm{~mm}$ glucose, consistent with the inhibition of monocarboxylate, but not glucose, transport. Similarly, 4-CIN suppressed EPSPs supported by $10 \mathrm{~mm}$ lactate within $30 \mathrm{~min}$ (EPSP slopes, $3.8 \pm 2.0 \% ; n=4$ ), but not EPSPs supported by 10 mM glucose (EPSP slopes, $96.0 \pm 2.0 \% ; n=4$ ) (Fig. 2). In contrast, EPSPs supported by $10 \mathrm{~mm}$ glucose (glucose-supported EPSPs) were depressed slowly by administration of CCB. Similar to glucose deprivation, it took $60 \mathrm{~min}$ for complete depression of glucose-supported EPSPs (Fig. 3; EPSP slopes, $69.6 \pm 6.9 \%$ at 20 min after administration; $n=5$ ). After complete depression of glucose-supported EPSPs by CCB, administration of $10 \mathrm{~mm}$ pyruvate for $30 \mathrm{~min}$ partially restored synaptic transmission (36.3 \pm $6.8 \%$ of control $30 \mathrm{~min}$ after pyruvate administration), suggesting that $\mathrm{CCB}$ has relative selectivity for glucose transporters. Similarly, $10 \mathrm{~mm}$ lactate administered $60 \mathrm{~min}$ after $\mathrm{CCB}$ restored EPSPs to $36.7 \pm 6.3 \%$ of control $(n=4)$.

\section{4-CIN and synaptic depression by glucose deprivation or CCB}

Although glucose-supported EPSPs were not depressed by 4-CIN (filled circles in Fig. 3; EPSP slope, $95.0 \pm 3.0 \%$ of control $20 \mathrm{~min}$ after 4 -CIN; $n=5$ ), administration of CCB in the presence of 4-CIN abolished glucose-supported EPSPs rapidly $(5.6 \pm 1.0 \%$ of control $20 \mathrm{~min}$ after $\mathrm{CCB} ; p<0.01$ as compared with CCB alone). Similarly, the decay of glucosesupported EPSPs during glucose deprivation was accelerated by pretreatment of slices with 4-CIN ( filled circles in Fig. $4 c$; EPSP slope, $6.9 \pm 2.5 \% 20$ min after glucose deprivation; $p<$ 0.01 as compared with glucose deprivation alone). In slices treated with 4-CIN, the depression of EPSPs by $60 \mathrm{~min}$ of glucose deprivation was poorly reversible after reintroduction of glucose $(7.9 \pm 2.3 \%$ of control; $p<0.01$ as compared with recovery in the absence of 4 -CIN). These results suggest that monocarboxylates participate in the maintenance of synaptic transmission and integrity during glucose deprivation.

\section{CCB and synaptic depression by pyruvate deprivation}

Similar to glucose-supported EPSPs during glucose deprivation, pyruvate-supported EPSPs show a slow depression during pyruvate deprivation (Fig. $5 c$; EPSP slope, $81.3 \pm 14.3 \%$ of control $20 \mathrm{~min}$ after pyruvate deprivation; $n=5$ ). If endogenous glucose from glia fuels neurons during energy source deprivation, the depression of pyruvate-supported EPSPs should occur more rapidly during pyruvate deprivation in the presence of CCB. We found that CCB had little effect on the depression of pyruvate-supported EPSPs ( filled circles in Fig. $5 c ; 76.5 \pm 13.2 \%$ of control $20 \mathrm{~min}$ after pyruvate deprivation in the presence of $\mathrm{CCB} ; n=5)$. Moreover, $\mathrm{CCB}$ had little effect on the recovery of EPSPs after reperfusion of $10 \mathrm{~mm}$ pyruvate after pyruvate deprivation $(84.9 \pm 7.1 \%$ vs $75.6 \pm$ $5.4 \%$ of control $30 \mathrm{~min}$ after pyruvate administration in the presence vs absence of CCB). These observations suggest that endogenous glucose is unlikely to participate in energy buffering in slices fueled by pyruvate. The depression of pyruvatesupported EPSPs during pyruvate deprivation was significantly faster when EPSPs had been suppressed previously by $60 \mathrm{~min}$ of glucose deprivation and then restored by $10 \mathrm{~mm}$ pyruvate (Fig. $5 d ; 7.1 \pm 1.0 \%$ of control $20 \mathrm{~min}$ after pyruvate deprivation; $n=4 ; p<0.01$ as compared with simple pyruvate deprivation as shown in the open circles in Fig. 5c). These observations, together with the finding that monocarboxylates do not support glycogenesis in the absence of glucose (Dringen 


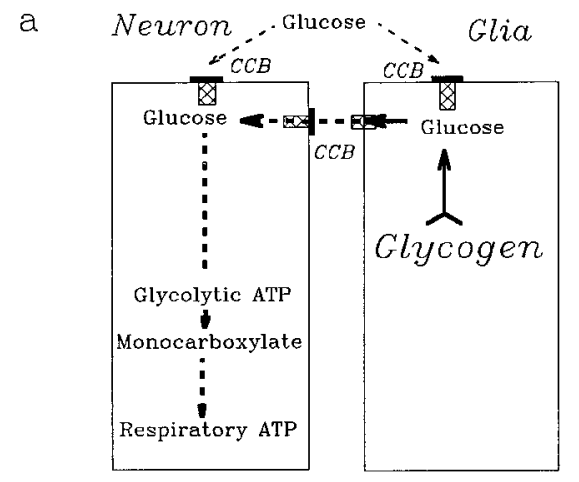

$\mathrm{b}$

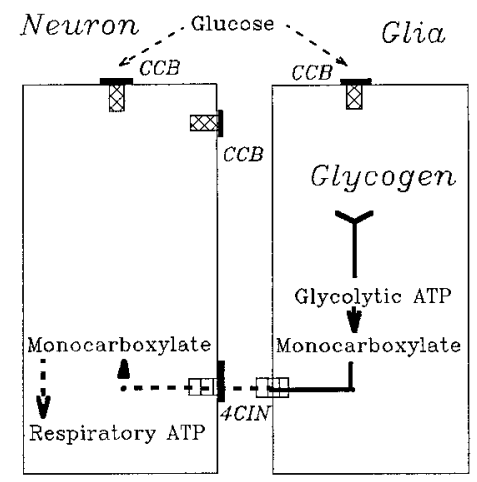

C

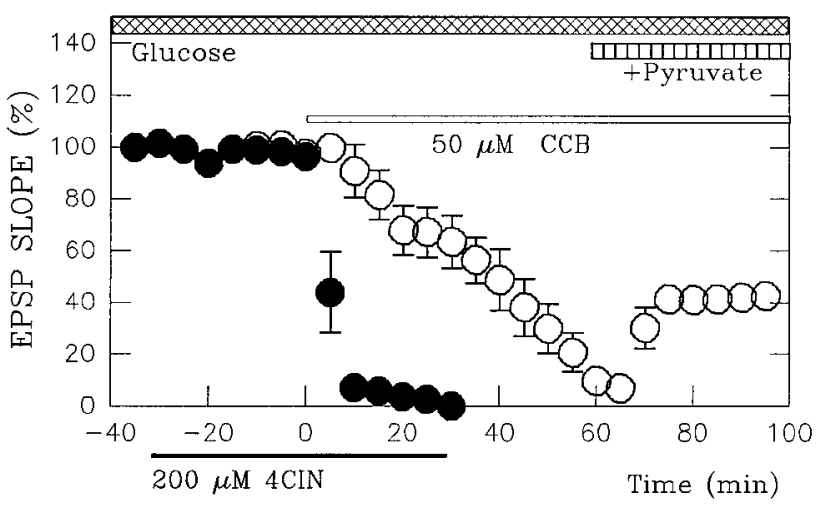

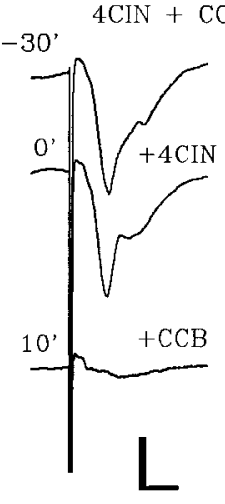

CCB alone

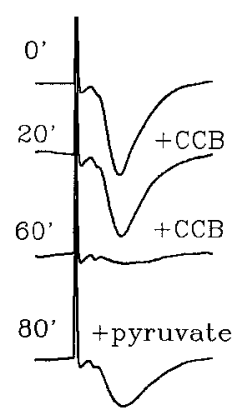

Figure 3. 4-CIN accelerates the suppression of glucose-supported EPSPs by CCB. $a$, Shown is a scheme in which endogenous glucose from glia fuels neurons. In this case $\mathrm{CCB}$ should be more effective than exogenous glucose deprivation, because the energy buffering system also is blocked by CCB. $b$, Shown is a scheme in which endogenous monocarboxylates from glia fuel neurons. The effects of CCB should be identical to glucose deprivation when endogenous sugars are not involved in energy buffering. However, the block of monocarboxylate transporters by 4-CIN should enhance the effects of CCB. $c$, Glucose-supported EPSPs (open circles) are slowly suppressed by $50 \mu \mathrm{M}$ CCB (open bar) and are partially restored by adding pyruvate (vertically striped bar). Glucosesupported EPSPs ( filled circles) are not depressed by $200 \mu \mathrm{M} 4$-CIN ( filled bar below the time axis) but are abolished promptly by CCB (open bar) in the presence of 4-CIN. The traces on the right show field EPSPs denoted with the time (in minutes) when the responses were obtained in the graph at left. Calibration bar, 1 $\mathrm{mV}, 5 \mathrm{msec}$. et al., 1993b), suggest that glycogen serves as an energy buffer during endogenous energy source deprivation.

\section{CCB and synaptic depression by lactate deprivation}

Similar to the findings with pyruvate, CCB had little effect on the depression of lactate-supported EPSPs (Fig. 6c). The depression of lactate-supported EPSPs during $20 \mathrm{~min}$ of lactate deprivation did not differ in the absence (open circles in Fig. $6 c$; $51.4 \pm 12.1 \%$ of control; $n=5$ ) or presence of CCB (closed circles in Fig. $6 c ; 46.5 \pm 7.3 \% ; n=5)$. Furthermore, CCB had little effect on the recovery of EPSPs after reperfusion of lactate after lactate deprivation $(78.6 \pm 3.7 \%$ vs $71.3 \pm 12.4 \%$ of control $30 \mathrm{~min}$ after lactate administration in the presence vs absence of CCB). When EPSPs had been suppressed previously by 60 min of glucose deprivation and then restored by 10 $\mathrm{mm}$ lactate, lactate deprivation promptly abolished lactatesupported EPSPs (Fig. $6 d ; 4.6 \pm 1.9 \%$ of control 20 min after lactate deprivation; $n=4 ; p<0.05$ as compared with simple lactate deprivation as shown in the open circles in Fig. 6c). These observations suggest again that glycogen serves as an energy buffer during energy deprivation but that endogenous glucose is unlikely to participate in energy buffering.

\section{Morphological effects of monocarboxylate transport inhibition}

The poor recovery of EPSPs when monocarboxylate transport is inhibited during glucose deprivation (Fig. 4c) could result from irreversible damage to slices. To examine this possibility, we studied the effects of the transport inhibitors on the morphology of CA1 neurons. When administered alone, neither $200 \mu \mathrm{M} 4$ CIN nor $50 \mu \mathrm{M}$ CCB produced significant neuronal damage during $90 \mathrm{~min}$ of administration (Fig. 7a,c; damage scores, $0.8 \pm$
$0.2, n=11$, and $1.2 \pm 0.5, n=5$, for 4 -CIN and CCB, respectively). However, the combination of 4-CIN with CCB produced clear injury to the CA1 pyramidal cell layer, resulting in dark cell appearances and neuronal dissolution (Fig. $7 b$; damage score, $2.6 \pm 0.2, n=13 ; p<0.01$ vs 4 -CIN or CCB alone). Similar changes were observed when glucose was removed from the media for $120 \mathrm{~min}$ or longer (Izumi et al., 1994) (Fig. 7f; damage score, $2.5 \pm 0.3, n=4$ ), but not during $90 \mathrm{~min}$ of glucose deprivation (Fig. $7 e$; damage score, $1.3 \pm 0.3, n=8$ ). In contrast, glucose deprivation plus $200 \mu \mathrm{M} 4-\mathrm{CIN}$ produced significant changes within $90 \mathrm{~min}$ (Fig. $7 d$; damage score, $2.6 \pm 0.3, n=10$; $p<0.05$ vs glucose deprivation alone; $p<0.01$ vs 4 -CIN alone). These results indicate that endogenous monocarboxylates play an important role in maintaining morphological integrity during energy source deprivation.

\section{Endogenous monocarboxylates and partial glucose deprivation}

To assess the role of monocarboxylates during less severe glucose reduction, we decreased extracellular glucose from 10 to $2 \mathrm{~mm} 30 \mathrm{~min}$ before administration of 4-CIN. As reported previously (Fleck et al., 1993; Izumi and Zorumski, 1997), the reduction of glucose to $2 \mathrm{~mm}$ did not alter baseline EPSPs (Fig. $8 a ; 95.8 \pm 1.7 \%$ of control; $n=5$ ). However, administration of $200 \mu \mathrm{M} 4-\mathrm{CIN}$ in the presence of the lower glucose level depressed EPSPs (64.2 $\pm 3.7 \%$ of control 20 min after 4 -CIN). EPSPs were restored to baseline in the presence of $2 \mathrm{~mm}$ glucose by removal of 4 -CIN (100.4 $\pm 6.6 \%$ of control after a 30 min washout). A similar depression of EPSPs by 4-CIN was seen in the presence of $3.3 \mathrm{~mm}$ glucose $(63.2 \pm 11.4 \%$ of control; $n=5$ ) with full recovery after washout of 4-CIN 


\begin{abstract}
Figure 4. 4-CIN accelerates the suppression of glucose-supported EPSPs by glucose deprivation. $a$, Shown is a scheme in which endogenous glucose from glia, but not monocarboxylates, fuels neurons during exogenous glucose deprivation. In this case 4-CIN should not affect the energy buffering system. $b$, Shown is a scheme in which endogenous monocarboxylates from glia fuel neurons during exogenous glucose deprivation. In this case 4CIN should worsen the effects of exogenous glucose deprivation. $c$, Administration of $200 \mu \mathrm{M} 4-\mathrm{CIN}$ ( filled bar below the time axis) does not depress glucose-supported EPSPs ( filled circles; time $-30-0 \mathrm{~min})$. Although glucose deprivation (open bar) normally takes longer than $20 \mathrm{~min}$ to abolish EPSPs (open circles; time 0-60 min; also see Fig. 2), glucose deprivation promptly depresses EPSPs during 4-CIN administration (filled circles; time $0-90 \mathrm{~min}$ ). The traces on the right are field EPSPs obtained before and 30 min after 4-CIN administration, 20 min after glucose deprivation in the presence of 4-CIN, and $30 \mathrm{~min}$ after readministration of glucose. The number on each trace denotes the time (in minutes) when the response was obtained in the graph at left. Calibration bar, $1 \mathrm{mV}, 5$ msec.
\end{abstract}

a

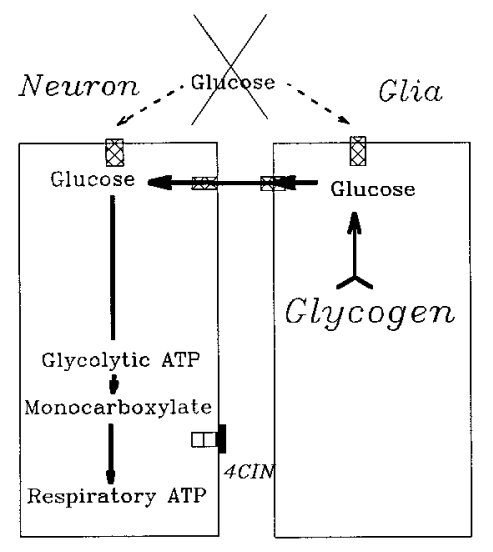

b

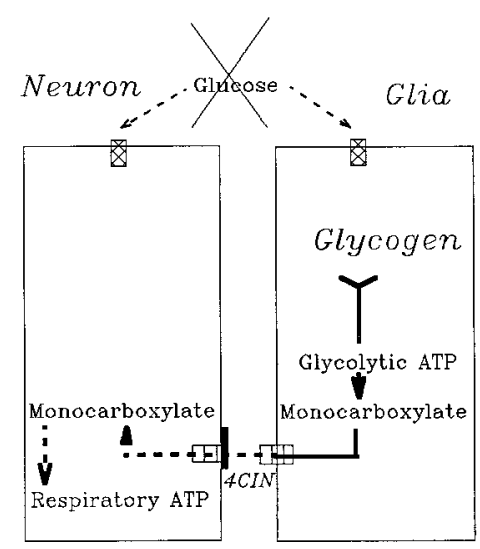

C

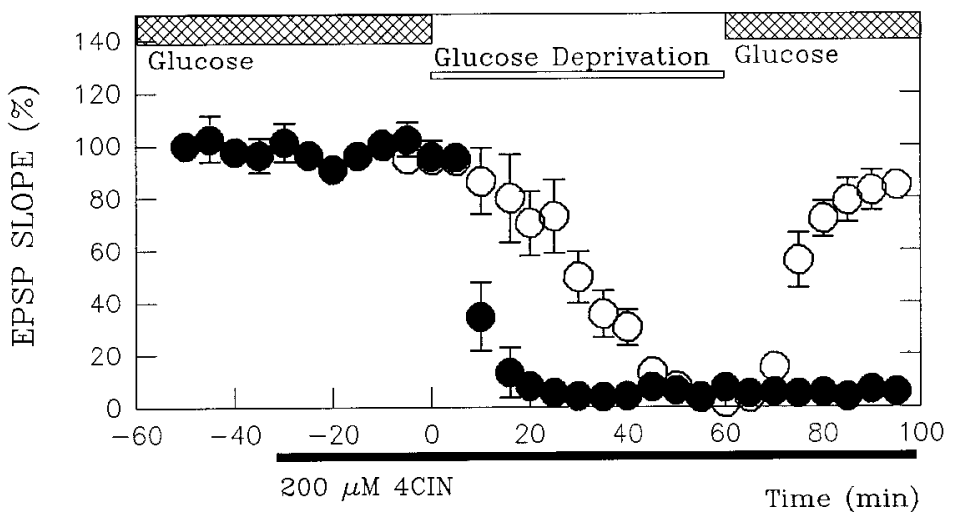

Glucose Deprivation

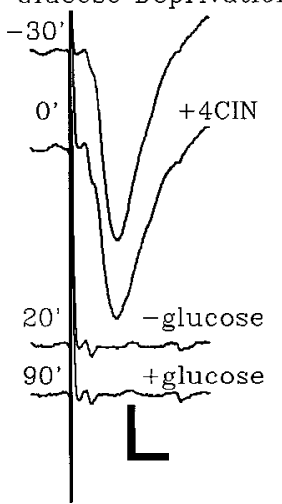

(99.1 $\pm 6.7 \%$ of control). Furthermore, in slices pretreated with 4-CIN a decrease in glucose from 10 to $2 \mathrm{~mm}$ resulted in depression of EPSPs (Fig. $8 b ; 33.5 \pm 8.9 \%$ of control $20 \mathrm{~min}$ after perfusion of $2 \mathrm{~mm}$ glucose; $n=5$ ). After restoration of 10 $\mathrm{mm}$ glucose, EPSPs returned to $86.3 \pm 7.9 \%$ of control. These results provide strong evidence that even during partial glucose deprivation endogenous monocarboxylates help to support synaptic transmission in the hippocampus.

\section{DISCUSSION}

Differences in the rate of synaptic depression during glucose deprivation and IA administration suggest the importance of an endogenous energy buffering system that depends on glycolysis to maintain synaptic function. This contention is supported by marked differences in the degree of neuronal damage produced by 90 min IA administration as compared with 90 min glucose deprivation (Izumi et al., 1994). Glycogen is believed to be a major source of such energy buffering (Swanson, 1992) and helps to sustain neurons during glucose deprivation in cortical cultures (Swanson and Choi, 1993). Because glycogen is stored primarily in glia and little is present in neurons (Rosenberg and Dichter, 1985; Kato et al., 1989; Ignacio et al., 1990), the energy buffering system must use substrates that can cross cell membranes to fuel neurons. High-energy phosphates, including ATP, are relatively impermeant to cell membranes. This makes it likely that glia fuel neighboring neurons by providing sugars or diffusible monocarboxylate glycolytic intermediates.

It is important to determine which agents form the buffering system, because sugars and monocarboxylates have reciprocal advantages and disadvantages as energy sources. For several functions, including ionic pumping, glycolytic energy may be preferred over energy derived oxidatively from lactate (Dirks et al., 1980; Winkler, 1981; Lipton and Robacker, 1983; Raffin et al., 1992; Swanson, 1992; Stittsworth and Lanthorn, 1993). However, the requirement for phosphorylation makes glucose use expensive under conditions in which ATP levels are limited. In contrast, lactate and pyruvate fuel neurons only when cellular respiration is operational.

Both glucose and the monocarboxylates can be released into the media of glial cultures, making it difficult to determine which energy substrate form is responsible for fueling neurons during energy source deprivation. In astroglia-rich primary cultures, Forsyth et al. (1996) detected 2-deoxyglucose extracellularly when astroglia were preloaded with labeled 2-deoxyglucose-6phosphate, whereas Dringen et al. (1993a) detected the release of lactate, but not glucose, from cultured glia. Astroglia also release pyruvate extracellularly in amounts that are sufficient to support neuronal growth (Selak et al., 1985). It is important to note that these experiments were done in neonatal cultures and that glucose use is limited in neonatal brain (Nehlig and De Vasconcelos, 1993) probably because of the low activity of hexokinase (Clark et al., 1993) and glucose transporters (Vannucci et al., 1993). Thus, the monocarboxylates rather than glucose may be the preferred energy substrates during early periods of development (Vicario et al., 1991; Young et al., 1991).

In hippocampal slices from 30-d-old rats, we used 4-CIN and $\mathrm{CCB}$ to examine whether glucose or the monocarboxylates main- 
a

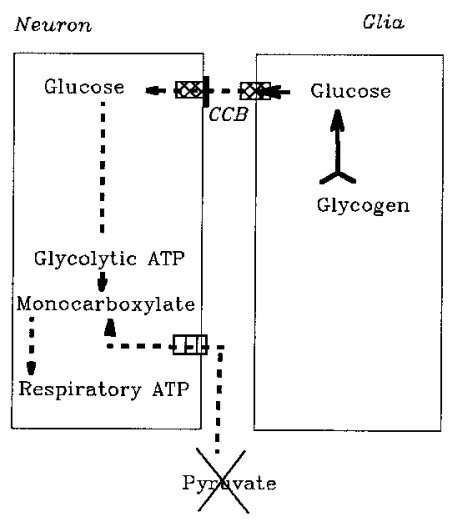

C

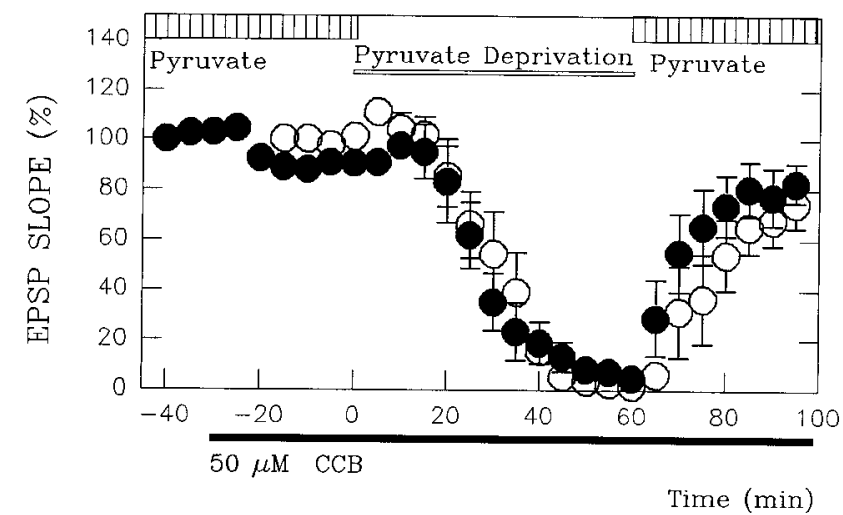

$\mathrm{d}$

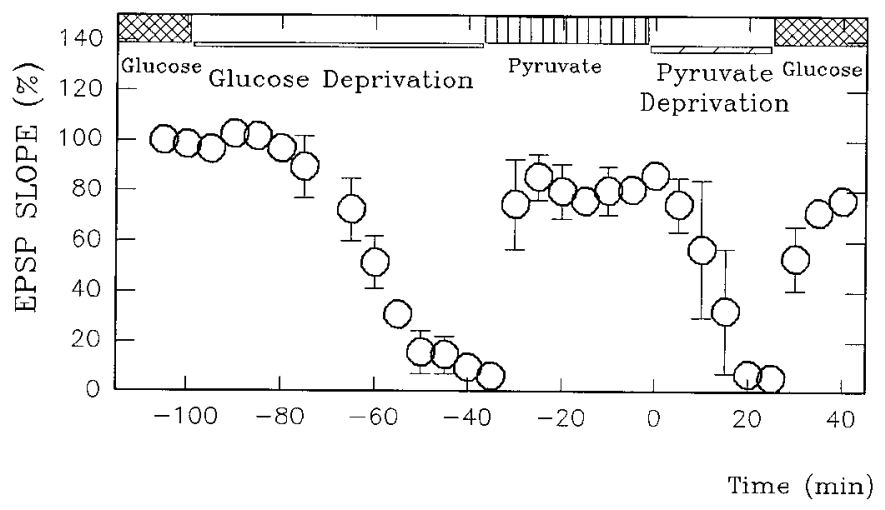

$\mathrm{b}$

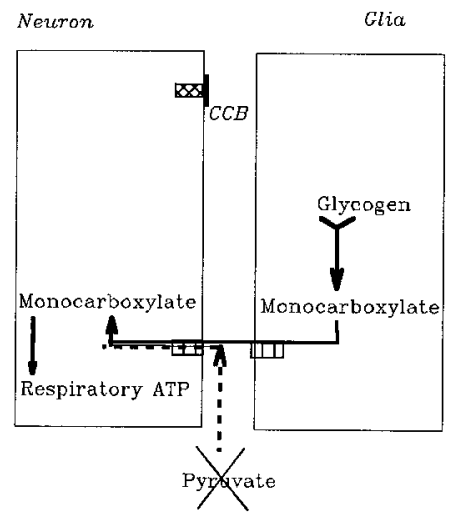

Figure 5. Effects of $\mathrm{CCB}$ on pyruvate-supported EPSPs. $a$, Shown is a scheme in which endogenous glucose from glia fuels neurons during exogenous pyruvate deprivation. CCB should induce total energy loss in neurons by blocking energy buffering. $b$, Depicted is a scheme in which endogenous monocarboxylates from glia fuel neurons during exogenous pyruvate deprivation. In this case CCB should not worsen the effects of exogenous pyruvate deprivation. $c$, EPSPs (open circles) supported by $10 \mathrm{~mm}$ pyruvate (vertically striped bar) instead of glucose are slowly depressed by pyruvate deprivation (open bar). Administration of $50 \mu \mathrm{M}$ CCB (solid bar below the time axis) neither accelerates the depression of EPSPs during pyruvate deprivation nor hampers the recovery during readministration of pyruvate (filled circles). The traces are representative field EPSPs with (right column) or without CCB. The number on each trace depicts the time when the response was obtained in the graph at left. Calibration bar, $1 \mathrm{mV}, 5$ msec. $d$, EPSPs are restored by $10 \mathrm{~mm}$ pyruvate after $60 \mathrm{~min}$ of glucose deprivation. This treatment accelerates the depression of pyruvate-supported EPSPs during pyruvate deprivation. The traces on the right are representative field EPSPs before and 20 and 60 min after glucose deprivation, 30 min after pyruvate administration (time $0^{\prime}$ ), $20 \mathrm{~min}$ after pyruvate deprivation (time $20^{\prime}$ ), and $20 \mathrm{~min}$ after glucose readministration. Calibration bar, $1 \mathrm{mV}, 5 \mathrm{msec}$. tain neuronal function and morphology during energy source deprivation. Fowler (1993) has shown that CCB can be used as a relatively selective glucose transport inhibitor in hippocampal slices. Although 4-CIN was developed initially as an inhibitor of mitochondrial pyruvate uptake (Halestrap and Denton, 1974; Halestrap, 1975), this does not seem to be the predominant action of 4-CIN in hippocampal slices, because both EPSPs and ATP levels are sustained in the presence of $500 \mu \mathrm{M} 4-\mathrm{CIN}$ plus $10 \mathrm{~mm}$ glucose (Cox et al., 1985), and other studies have shown that 4-CIN is an effective monocarboxylate transport inhibitor (Garcia et al., 1995; Williams et al., 1996).

Consistent with selective effects on monocarboxylate transport, we found that 4-CIN rapidly depressed both pyruvate- and lactate-supported EPSPs but failed to alter glucose-supported EPSPs. In contrast, CCB produced only a slow depression of glucose-supported EPSPs that was similar in time course to simple glucose deprivation and failed to alter the time course of depression of pyruvate-supported EPSPs during pyruvate deprivation. The slow time course of synaptic depression in slices treated with $\mathrm{CCB}$ did not result from slow actions of the drug, because administration of CCB to slices pretreated with 4-CIN led to a prompt suppression of synaptic responses. When administered during glucose deprivation or during CCB administration, 4-CIN caused a rapid decline in synaptic responsiveness and morphological neuronal integrity. These observations strongly suggest that energy substrates that use a 4-CIN-sensitive transporter play a major role in maintaining neuronal function during energy source deprivation.

The ability to use monocarboxylates as an energy source requires oxidative metabolism and is severely compromised 
a

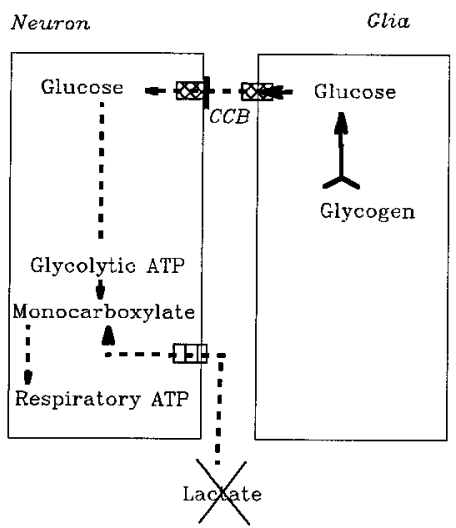
supported EPSPs. a, Shown is a scheme in which endogenous glucose from glia fuels neurons during exogenous lactate deprivation. CCB should induce total energy loss in neurons by blocking energy buffering. $b$, Depicted is a scheme in which endogenous monocarboxylates from glia fuel neurons during exogenous lactate deprivation. In this case $\mathrm{CCB}$ should not worsen the effects of exogenous lactate deprivation. $c$, EPSPs (open circles) supported by $10 \mathrm{~mm}$ lactate (vertically striped bar) instead of glucose are slowly depressed by lactate deprivation (open bar). Administration of $50 \mu \mathrm{M}$ CCB (solid bar below the time axis) neither accelerates the depression of EPSPs during lactate deprivation nor hampers the recovery during readministration of lactate ( filled circles). The traces are representative field EPSPs with (right column) or without CCB. The number on each trace depicts the time when the response was obtained in the graph at left. Calibration bar, $1 \mathrm{mV}, 5 \mathrm{msec}$. $d$, EPSPs are restored by $10 \mathrm{~mm}$ lactate (vertically striped bar) after $60 \mathrm{~min}$ of glucose deprivation. This treatment accelerates the depression of lactatesupported EPSPs during pyruvate deprivation. The traces on the right are representative field EPSPs before and 20 and 60 min after glucose deprivation, 30 min after lactate administration (time $0^{\prime}$ ), $20 \mathrm{~min}$ after lactate deprivation (time $20^{\prime}$ ), and $20 \mathrm{~min}$ after glucose readministration. Calibration bar, $1 \mathrm{mV}, 5 \mathrm{msec}$.

C $\mathrm{b}$
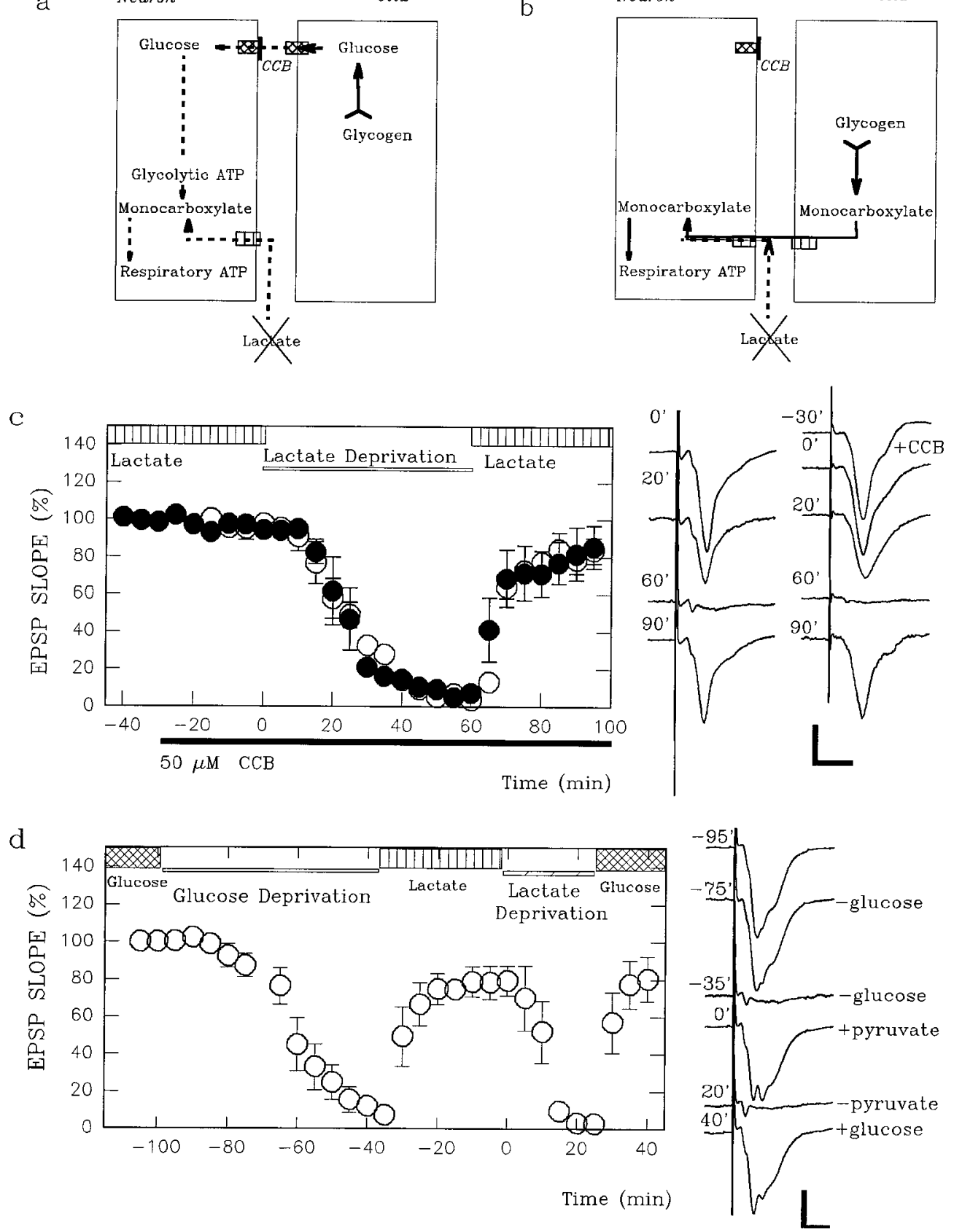

during anoxia. Although this requirement for oxidative metabolism may be disadvantageous under certain circumstances, monocarboxylate use, in contrast to glycolysis, does not require initial energy expenditure to drive ATP production and can function when glycolysis is inhibited. The latter situation may be particularly important when glycolytic enzymes are inhibited by aldehydes derived from ketone body use (Novotny et al., 1994). It is also important to consider whether neurons and glia have different requirements for glucose and whether there are differential uses of ATP produced from glycolysis versus respiration. Several lines of evidence suggest that ionic pumps are fueled preferentially by glycolytic ATP (Lipton and Robacker, 1983; Raffin et al., 1992) and that glucose is required to maintain glial energy levels (Kauppinen et al., 1988). Interestingly, pyruvate seems to be unable to substitute for glucose in maintaining glial functions that require ATP consumption (Kauppinen et al., 1988).

Our data also indicate that monocarboxylates help to maintain synaptic function during periods of hypoglycemia. During mild glucose deprivation (2-3.3 mm glucose) baseline synaptic responses were unaltered, yet 4-CIN caused a depression of EPSPs that was reversed either by drug washout or by restoration of higher glucose levels. These observations suggest that the glialneuronal energy buffering system is important even at $2-3.3 \mathrm{~mm}$ glucose. Although glycogen storage depends on extracellular glucose levels (Passonneau and Crites, 1976) and glycogen stores can be depleted rapidly by glucose deprivation (Siesjo, 1978), the stability of synaptic transmission in the presence of $2 \mathrm{~mm}$ glucose suggests that even this level of extracellular glucose is sufficient to allow glia to support basal neuronal activity for a prolonged 


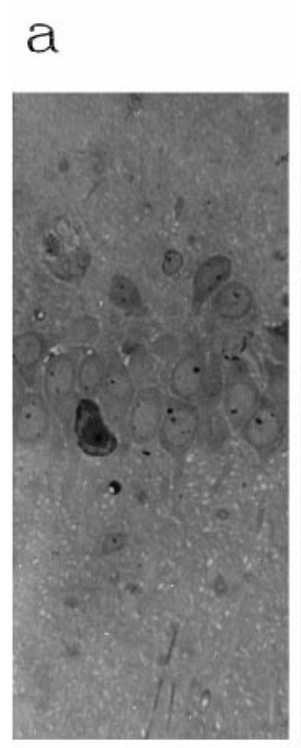

b

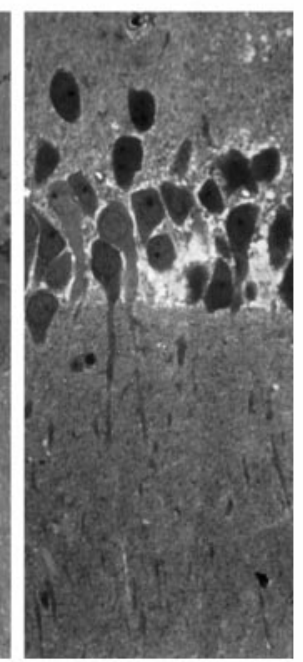

$\mathrm{C}$

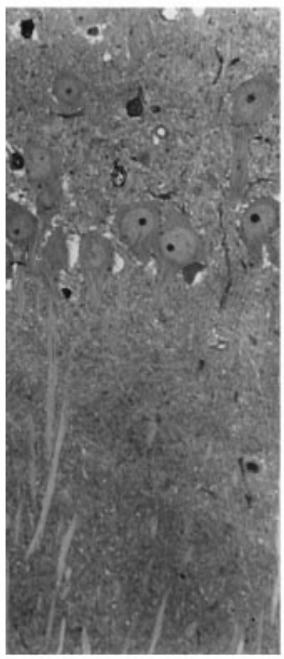

$\mathrm{d}$

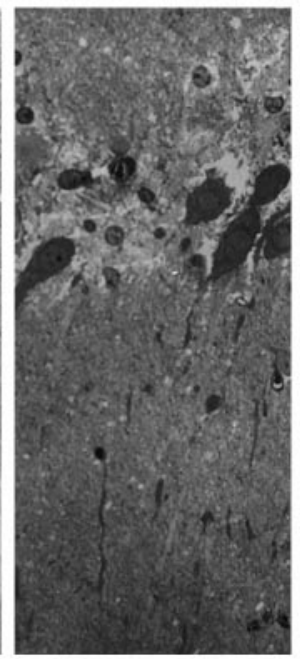

e

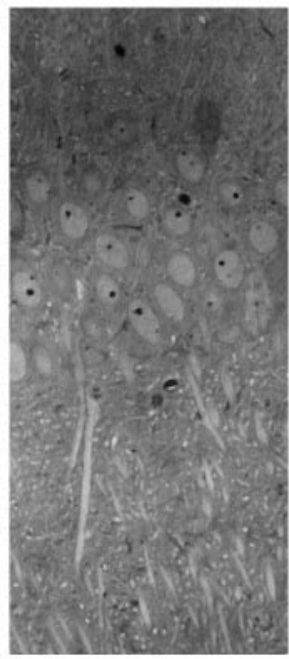

$\mathrm{f}$

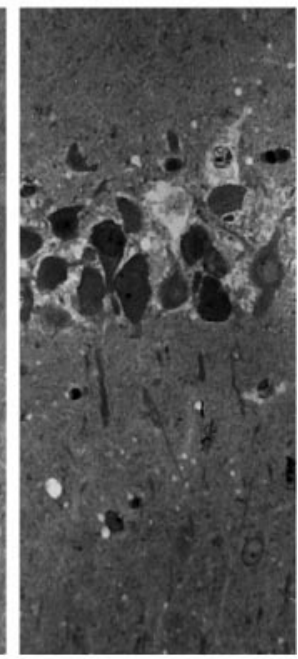

Figure 7. Morphological disintegration of pyramidal cells by 4-CIN and CCB. Slices were stained with methylene blue and azure II. $a$, After 90 min incubation with $200 \mu \mathrm{M}$ 4-CIN alone, the CA1 region of hippocampal slices appears intact except for one dark cell in the middle (damage score was rated as 1 for this slice). $b, c$, Although in the presence of $50 \mu \mathrm{M} \mathrm{CCB}$ the pyramidal cells show some changes $(c)$, dark cells and dissolution of the cell body layer are much more prominent after incubation with both CCB and 4-CIN $(b)$. Damage scores for these two slices were 3 and 1 for $b$ and $c$, respectively. $d$, $e$, Similar severe degeneration is produced by $200 \mu \mathrm{M} 4$-CIN in the absence of glucose for 90 min $(d)$, although 90 min of glucose deprivation alone fails to produce morphological changes $(e)$. Damage scores were 3 and 0 for $d$ and $e$, respectively. $f$, Prolonged glucose deprivation for 180 min causes dark cell changes in CA1 pyramidal neurons $(f)$. Damage score for this panel was 3. Magnification, 320×; scale bar, $20 \mu \mathrm{m}$.

period via monocarboxylate release. Because of its rapid and continuous turnover (Watanabe and Passonneau, 1973), glial glycogen has been proposed to play a dynamic role both as an energy source supporting basal neuronal function and as an emergency energy reserve (Swanson, 1992). Continuous involvement of glial metabolism in supporting neuronal function has been observed in the retina where Müller cells sustain photoreceptor activity via lactate (Poitry-Yamate et al., 1995). Additionally, Bittar et al.
(1996) have shown that lactate dehydrogenase-5 (LDH-5), an isoform that favors lactate production, is found in astroglia, whereas LDH-1, an isoform that favors lactate use, is found in neurons.

In summary, the present study provides strong support for the idea that monocarboxylates rather than glucose are the primary source that glia use to fuel neurons in the hippocampus during energy depletion. However, it is important to note that the
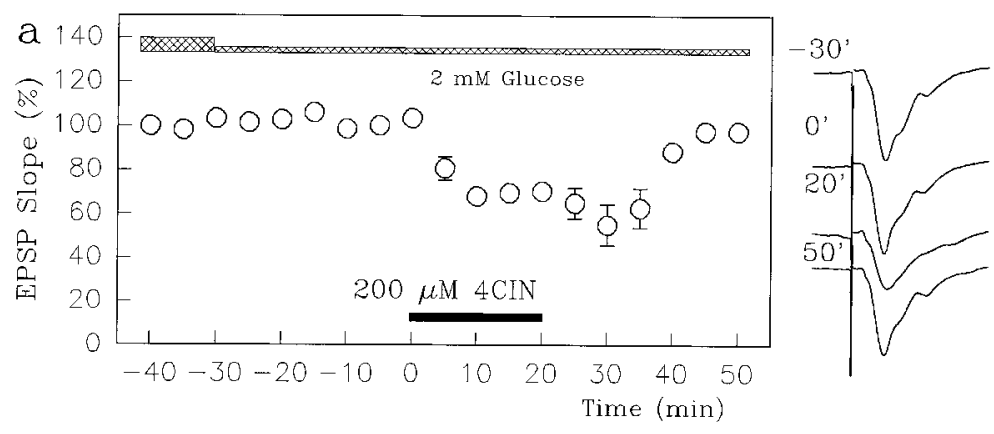

Figure 8. EPSPs supported by $2 \mathrm{~mm}$ glucose, but not by $10 \mathrm{~mm}$ glucose, are sensitive to 4-CIN. $a$, Although changing the glucose concentration from $10 \mathrm{~mm}$ to $2 \mathrm{~mm}$ does not alter baseline EPSPs (Izumi and Zorumski, 1997), administering $200 \mu \mathrm{M}$ 4-CIN (filled bar) in the presence of $2 \mathrm{~mm}$ glucose depresses EPSPs. EPSPs recover after drug washout even in the presence of $2 \mathrm{~mm}$ glucose. Representative EPSPs in the presence of 10 $\mathrm{mm}$ glucose, $30 \mathrm{~min}$ after reducing the glucose concentration to $2 \mathrm{mM}$ (time 0), $20 \mathrm{~min}$ after administration of 4-CIN, and 30 min after washout (time 50) are shown to the right of the graphs. $b$, Administration of $200 \mu \mathrm{M} 4$-CIN ( filled bar) does not depress EPSPs supported by $10 \mathrm{~mm}$ glucose (see Figs. 4, 5). However, lowering the glucose concentration to $2 \mathrm{mM}$ for $30 \mathrm{~min}$ (time 0-30) depresses EPSPs in the presence of 4-CIN. This depression is reversed by reperfusion with $10 \mathrm{~mm}$ glucose (time $30-50)$. The traces depict representative field EPSPs before and 30 min after administration of 4-CIN (time -30 and 0 ), $20 \mathrm{~min}$ after lowering glucose concentration, and $20 \mathrm{~min}$ after returning the glucose concentration to $10 \mathrm{~mm}$ (time 50). Calibration bar, $1 \mathrm{mV}, 5 \mathrm{msec}$. 
present experiments were performed at $30^{\circ} \mathrm{C}$ in slices prepared from young animals. Because metabolic and transport processes could differ at higher temperatures and older ages, future studies should examine the effects of these variables on monocarboxylate use. Additionally, monocarboxylate transporters are shared by lactate, pyruvate, and ketone bodies, and pyruvate is converted promptly to lactate (Wolfe, 1990). Thus, future studies also should address changes in glycogen, lactate, and pyruvate levels in slices and whether lactate, pyruvate, or other monocarboxylates play the major role in preserving neuronal function during periods of low energy supply.

\section{REFERENCES}

Bhattacharya SB, Datta AG (1993) Is brain a gluconeogenic organ? Mol Cell Biochem 125:51-57.

Bittar PG, Charnay Y, Pellerin L, Bouras C, Magistretti PJ (1996) Selective distribution of lactate dehydrogenase isoenzymes in neurons and astrocytes of human brain. J Cereb Blood Flow Metab 16:1079-1089.

Cambray-Deakin M, Pearce B, Morrow C, Murphy S (1988) Effects of neurotransmitters on astrocyte glycogen stores in vitro. J Neurochem 51:1852-1857.

Clark JB, Bates TE, Cullingford T, Land JM (1993) Development of enzymes of energy metabolism in the neonatal mammalian brain. Dev Neurosci 15:174-180.

Cox DWG, Drower J, Bachelard HS (1985) Effects of metabolic inhibitors on evoked activity and energy state of hippocampal slices perfused in vitro. Exp Brain Res 57:464-470.

Dirks B, Hanke J, Kriegelstein J, Stock R, Wickop G (1980) Studies on the linkage of energy metabolism and neuronal activity in the isolated perfused rat brain. J Neurochem 35:311-317.

Dringen R, Hamprecht B (1992) Investigation on the function of glycogen in astrocytes. Biol Chem Hoppe-Seyler 373:951.

Dringen R, Gebhardt R, Hamprecht B (1993a) Glycogen in astrocytes: possible function as lactate supply for neighboring cells. Brain Res 623:208-214.

Dringen R, Schmoll D, Cesar M, Hamprecht B (1993b) Incorporation of radioactivity from $\left[{ }^{14} \mathrm{C}\right]$ lactate into the glycogen of cultured mouse astroglial cells. Evidence for gluconeogenesis in brain cells. Biol Chem Hoppe-Seyler 374:343-347.

Dringen R, Wiesinger H, Hamprecht B (1993c) Uptake of L-lactate by cultured rat brain neurons. Neurosci Lett 163:5-7.

Fleck MW, Henze DA, Barrionuevo G, Palmer AM (1993) Aspartate and glutamate mediate excitatory synaptic transmission in area CA1 of the hippocampus. J Neurosci 13:3944-3955.

Forsyth RJ (1996) Astrocytes and the delivery of glucose from plasma to neurons. Neurochem Int 28:231-241.

Forsyth RJ, Bartlett K, Burchell A, Scott HM, Eyre JA (1996) Dephosphorylation of 2-deoxyglucose-6-phosphate and 2-deoxyglucose export from cultured astrocytes. Neurochem Int 28:243-250.

Fowler JC (1993) Glucose deprivation results in a lactate preventable increase in adenosine and depression of synaptic transmission in rat hippocampal slices. J Neurochem 6:572-576.

Garcia CK, Brown MS, Pathak PK, Goldstein JL (1995) dDNA cloning of MCT2, a second monocarboxylate transporter expressed in different cells than MCT1. J Biol Chem 270:1843-1849.

Garriga J, Sust M, Cusso R (1994) Regional distribution of glycogen, glucose, and phosphorylated sugars in rat brain after intoxicating doses of ethanol. Neurochem Int 25:175-181.

Halestrap AP (1975) The mitochondrial pyruvate carrier. Kinetics and specificity of substrates and inhibitors. Biochem J 148:85-96.

Halestrap AP, Denton RM (1974) Specific inhibition of pyruvate transport in rat liver mitochondria and human erythrocytes by $\alpha$-cyano-4hydroxycinnamate. Biochem J 138:313-316.

Ignacio PC, Baldwin BA, Vijayan VK, Tait RC, Gorin FA (1990) Brain isozyme of glycogen phosphorylase: immunohistological localization within the central nervous system. Brain Res 529:42-49.

Izumi Y, Zorumski CF (1997) Involvement of nitric oxide in low glucose-mediated inhibition of hippocampal long-term potentiation. Synapse 25:258-262.

Izumi Y, Benz AM, Zorumski CF, Olney JW (1994) Effects of lactate and pyruvate on glucose deprivation in rat hippocampal slices. NeuroReport 5:617-620.

Izumi Y, Benz AM, Clifford DB, Zorumski CF (1996) Nitric oxide inhibitors attenuate ischemic degeneration in the CA1 rat hippocampal slices. Neurosci Lett 210:157-160.

Kato K, Shimizu A, Jurobe N, Takashi M, Koshikawa T (1989) Human brain-type glycogen phosphorylase: quantitative localization in human tissues determined with an immunoassay system. J Neurochem 52:1425-1432.

Kauppinen RA, Enkvist K, Holopainen I, Åkerman KEO (1988) Glucose deprivation depolarizes plasma membrane of cultured astrocytes and collapses transmembrane potassium and glutamate gradients. Neuroscience 26:283-289.

Lipton P, Robacker K (1983) Glycolysis and brain function: $\left[\mathrm{K}^{+}\right]_{\mathrm{o}}$ stimulation of protein synthesis and $\mathrm{K}^{+}$uptake require glycolysis. FASEB J 42:2875-2880.

Lowry OH, Passonneau JV, Hasselberger FH, Schulz DW (1964) Effect of ischemia on known substrates and cofactors of the glycolytic pathway in brain. J Biol Chem 239:18-30.

Magistretti PJ, Pellerin L (1996) Cellular bases of brain energy metabolism and their relevance to functional brain imaging: evidence for a prominent role of astrocytes. Cereb Cortex 6:50-61.

Maran A, Cranston I, Lomas J, MacDonald M, Amiel S (1994) Protection by lactate of cerebral function during hypoglycemia. Lancet 343:16-20.

Nehlig A, De Vasconcelos AP (1993) Glucose and ketone body utilization by the brain of neonatal rats. Prog Neurobiol 40:163-221.

Novotny MV, Yancey MF, Stuart R, Wiesler D, Peterson RG (1994) Inhibition of glycolytic enzymes by endogenous aldehydes: possible relation to diabetic neuropathies. Biochim Biophys Acta 1226:145-150.

Passonneau JV, Crites S (1976) Regulation of glycogen metabolism in astrocytoma and neuroblastoma cells. J Biol Chem 251:2015-2022.

Poitry-Yamate CL, Poity S, Tsacopoulos M (1995) Lactate released by Müller glial cells is metabolized by photoreceptors from mammalian retina. J Neurosci 15:5179-5191.

Prasannan KG, Subramanyam K (1968) Effects of insulin on the synthesis of glycogen in cerebral cortical slices of alloxan diabetic rats. Endocrinology 82:1-6.

Raffin CH, Rosenthal M, Busto R, Sick TJ (1992) Glycolysis: oxidative metabolism and brain potassium ion clearance. J Cereb Blood Flow Metab 12:34-42.

Rosenberg PA, Dichter MA (1985) Glycogen accumulation in rat cerebral cortex in dissociated cell culture. J Neurosci Methods 15:101-112.

Rust RS (1994) Energy metabolism of developing brain. Curr Opin Neurol 7:160-165.

Sagar SM, Sharp FR, Swanson RA (1987) The regional distribution of glycogen in rat brain fixed by microwave irradiation. Brain Res 417:172-174.

Schurr A, West CA, Rigor BM (1988) Lactate-supported synaptic function in the rat hippocampal slice preparation. Science 240:1326-1328.

Scrutton MC, Utter MF (1968) The regulation of glycolysis and gluconeogenesis in animal tissues. Annu Rev Biochem 3:249-302.

Selak I, Skaper SD, Varon S (1985) Pyruvate participation in the low molecular weight tropic activity for central nervous system neurons in glial-conditioned media. J Neurosci 5:23-28.

Siesjo BK (1978) Brain energy metabolism, 6. Metabolism of substrates in the brain, pp 151-209. New York: Wiley.

Sokoloff L, Rivitch M, Kennedy C, Des Rosiers MH, Patlak CS, Pettigrew KD, Sakurada O, Shinohara M (1977) The [14C]deoxyglucose method for the measurement of local cerebral glucose utilization; theory, procedure, and normal values in the conscious and anesthetized albino rat. J Neurochem 28:897-916.

Stittsworth Jr JD, Lanthorn TH (1993) Lactate mimics only some effects of D-glucose on epileptic depolarization and long-term synaptic failure. Brain Res 620:21-27.

Swanson RA (1992) Physiologic coupling of glial glycogen metabolism to neuronal activity in brain. Can J Physiol Pharmacol 70:S138-S144.

Swanson RA, Choi DW (1993) Glial glycogen stores affect neuronal survival during glucose deprivation in vitro. J Cereb Blood Flow Metab 13:162-169.

Tsacopoulos M, Magistretti PJ (1996) Metabolic coupling between glia and neurons. J Neurosci 16:877-885. 
Vannucci SJ, Willing LB, Vannucci RC (1993) Developmental expression of glucose transporters, Glut1 and Glut3, in postnatal rat brain. Adv Exp Med Biol 331:3-7.

Vicario C, Arizmendi C, Malloch G, Clark JB, Medina JM (1991) Lactate utilization by isolated cells from early neonatal brain. J Neurochem 57:1700-1707.

Walz W, Muckerji S (1988) Lactate release from cultured astrocytes and neurons: a comparison. Glia 1:366-370.

Watanabe H, Passonneau JV (1973) Factors affecting the turnover of cerebral glycogen and limit dextrin in vivo. $\mathrm{J}$ Neurochem 20:1543-1554.

Williams RJ, Maus M, Stella N, Glowinski J, Premont J (1996) Reduced glucose metabolism enhances the glutamate-evoked release of arachidonic acid from striatal neurons. Neuroscience 74:461-468.

Winkler BS (1981) Glycolytic and oxidative metabolism in relation to retinal function. J Gen Physiol 77:667-692.

Wolfe RR (1990) Isotopic measurement of glucose and lactate kinetics. Ann Med 22:163-170.

Young RSK, Petroff OAC, Chen B, Aquila Jr WJ, Gore JC (1991) Preferential utilization of lactate in neonatal dog brain: in vivo and in vitro proton NMR study. Biol Neonate 59:46-53.

Zorumski CF, Mennerick S, Izumi Y (1996) Assessment of synaptic effects of nitric oxide in hippocampal neurons. Methods Neurosci 31:283-299. 